\title{
Localization of Kaluza-Klein gauge fields on a brane.
}

\author{
Andrey Neronov * \\ Theoretische Physik, Universität Munchen, 80333, Munich, Germany
}

(October 22, 2018)

\begin{abstract}
In phenomenological models with extra dimensions there is a natural symmetry group associated to a brane universe, - the group of rotations of normal bundle of the brane. We consider Kaluza-Klein gauge fields corresponding to this group and show that they can be localized on the brane in models with warped extra dimensions. These gauge fields are coupled to matter fields which have nonzero rotation moment around the brane. In a particular example of a four-dimensional brane embedded into six-dimensional asymptotically anti-deSitter space, we calculate effective four-dimensional coupling constant between the localized fermion zero modes and the Kaluza-Klein gauge field.
\end{abstract}

*e-mail:neronov@theorie.physik.uni-muenchen.de 


\section{INTRODUCTION.}

A possibility to treat observable gauge fields as arising from dimensional reduction of higher-dimensional General Relativity has a long history [1]. If we suppose that the spacetime is a direct product of four-dimensional Minkowsky space $R^{4}$ with coordinates $x^{\mu}, \mu=$ $0, . ., 3$ on an $n$-dimensional "internal space" $K^{n}$ parameterized by coordinates $y^{a}, a=1, . ., n$, the four-dimensional gauge fields $A_{\mu}^{a}$ can be described by the $\mu a$ components of the higherdimensional metric

$$
g_{A B}=\left(\begin{array}{c|l}
g_{\mu \nu}+g_{a b} A_{\mu}^{a} A_{\nu}^{b} & g_{a b} A_{\mu}^{b} \\
\hline g_{a b} A_{\mu}^{b} & g_{a b}
\end{array}\right)
$$

In this simple form such an approach to the gauge interactions faces certain problems as, for example, the problem of obtaining realistic pattern of four-dimensional fermions with different charges with respect to $A_{\mu}^{a}$ after dimensional reduction of higher-dimensional fermion fields [2]. The problem of chiral fermions can be solved in models with additional fundamental higher-dimensional gauge fields fields in topologically nontrivial configurations [3], or in models with noncompact internal spaces 4 .

If we deal with noncompact internal spaces, we face a problem how to explain the observable four-dimensional structure of the universe. One is forced to consider the observable space-time as a surface (brane) embedded in a higher-dimensional manifold [5, [0]. The idea of the "brane world" received a considerable attention recently due to new developments in the string theory. A new impulse to higher-dimensional model building was given by papers [7,8] where the possibility of having "large" or infinite extra dimensions was considered in relation to the hierarchy problem of particle physics. The observational consequences of the brane world picture for the accelerator physics [9], astrophysics [10] and cosmology 11, 12 were extensively studied recently. The possibility of solving the fermion mass hierarchy [13], cosmological constant [14], supersymmetry breaking [15] problems with large extra dimensions was also discussed.

An important problem which must be addressed in phenomenological models with a brane universe is how the observable matter fields of the Standard Model of particle physics are localized on the brane. The mechanism of localization of fermions is known since a long time [16] while mechanisms of localization of gauge fields 17 20 were found recently.

In this paper we discuss the question whether it is possible to localize the Kaluza-Klein fields $A_{\mu}^{a}$ on the brane and to treat them as observable gauge gauge fields. If the brane is embedded in a space-time with $n \geq 2$ extra dimensions there is a "natural" symmetry group associated to the brane: the group of rotations of its normal bundle. In the case of $n=2$ extra dimensions the brane can be treated as a string-like defect in a higher-dimensional space-time. The symmetry group of the normal bundle is $U(1)$ and corresponds to rotations of normal vectors around the brane. We analyze the gauge field $A_{\mu}$ which corresponds to this symmetry in Kaluza-Klein Ansatz (1.1). We show that $A_{\mu}$ can be localized on a four-dimensional string even if the extra dimensions are noncompact and have infinite volume, as it is, for example, in the space-times considered in [6]. We consider coupling of the field $A_{\mu}$ to the localized fermion fields in a particular model where a four-dimensional brane is embedded into a six-dimensional asymptotically anti-deSitter space-time [21,22]. In this space-time both the Kaluza-Klein field $A_{\mu}$ which corresponds to the $U(1)$ symmetry of 
rotations of the normal bundle and the fermion zero modes which have nonzero momentum of rotation around the string are localized. We calculate the effective four-dimensional fine structure constant $\alpha$ in this model and find that it is related to the curvature radius of anti-deSitter space-time and to the thickness of the brane.

\section{GAUGE FIELDS ASSOCIATED TO THE GROUP OF ROTATIONS OF NORMAL BUNDLE.}

In the models with large or infinite extra dimensions the gauge fields $A_{\mu}^{a}$ which appear by dimensional reduction mechanism (1.1) are not considered as observable gauge fields of the Standard Model because of the following reasons. The Kaluza-Klein fields $A_{\mu}^{a}$ (1.1) are, in general, not confined to the brane and can propagate in the bulk. The tower of KaluzaKlein excitations of these fields starts from very low masses. For example, in the model with submillimeter extra dimensions [7] the mass scale of Kaluza-Klein excitations is of order of $10^{-3} \mathrm{eV}$ which means that there must be corrections to Coulomb law at distances of order of $1 \mathrm{~mm}$. In the case of noncompact extra dimensions one encounters another difficulty. Consider the case of single extra dimension. A four-dimensional brane $M^{4}$ is embedded in higher dimensional bulk $\mathcal{M}^{5}$ as a level surface of some function $F\left(x^{A}\right), A=0, . ., 5$

$$
M^{4}:\left\{F\left(x^{A}\right)=0\right\}
$$

If we take coordinates $x^{\mu}$ on the brane and the function $F$ as a coordinate system in the vicinity of the brane, the metric on $\mathcal{M}^{5}$ takes the form

$$
d s^{2}=g_{\mu \nu} d x^{\mu} d x^{\nu}+g_{44} d F^{2}
$$

since vector $\vec{N}_{A}=F_{, A}$ is a normal to the surfaces $F\left(x^{A}\right)=$ const. Comparing (2.2) with (I.1) we find that it is always possible to find a coordinate system in the neighborhood of the brane in which

$$
A_{\mu}\left(x^{A}\right) \equiv 0
$$

If the extra dimension is noncompact, such a coordinate system can be chosen globally and $A_{\mu}$ can be always removed by a gauge transformation of five-dimensional theory.

The situation changes if the number of extra dimensions is $n \geq 2$. In this case we have $4+n$ coordinate transformations

$$
x^{A} \rightarrow x^{A}+\xi^{A}(x)
$$

at our disposal. Therefore we can impose $4+n$ gauge conditions on the metric. For example, $g_{4 \mu}=0, g_{44}=1, g_{4 a}=0, a=1, . .,(n-1)$. After such a gauge fixing the metric becomes

$$
g_{A B}=\left(\begin{array}{c|c|c}
g_{\mu \nu}+g_{a b} A_{\mu}^{a} A_{\nu}^{b} & 0 & g_{a b} A_{\mu}^{b} \\
\hline 0 & 1 & 0 \\
\hline g_{a b} A_{\mu}^{b} & 0 & g_{a b}
\end{array}\right)
$$

The fields $A_{\mu}^{a}, a=1, . .,(n-1)$ can not be removed by a coordinate transformation. These fields have a clear geometrical meaning. Let the coordinate $r=x^{4}$ be a distance from 
the brane $M^{4}$ placed at $r=0$. Then the fields $A_{\mu}^{a}$ are the gauge fields associated to the symmetry group $G$ of rotations of the normal bundle of the brane. In the simplest case of a thin four-dimensional brane $M^{4}$ embedded into a higher-dimensional manifold $\mathcal{M}^{4+n}$ the coordinates $\theta^{a}, a=1, \ldots,(n-1)$ parameterize a small $(n-1)$-dimensional sphere around the point $r=0$ of location of the brane and the group of the normal bundle is $S O(n)$. The group $G$ may be more involved if we consider branes of nonzero thickness. The stress-energy tensor of the brane is different from zero in a region $M^{4} \times K^{n}=\{r \leq \epsilon\}$ for some small $\epsilon$. The surface $r=\epsilon$ is a $3+n$-dimensional boundary $M^{4} \times \partial K^{n}$ of the thick brane. The group $G$ is the group of isometries of $\partial K^{n}$. It can be quite arbitrary. For example, in the model considered in [14, this group is $S U(2) \times U(1)$.

If we introduce a coordinate system $\left(x^{\mu}, \theta^{a}\right)$ on $M^{4} \times \partial K^{n}$, the background metric (with $\left.A_{\mu}^{a}=0\right)$ in the vicinity of the brane can be written in the form

$$
d s^{2}=e^{\nu(r)} \eta_{\mu \nu} d x^{\mu} d x^{\nu}+e^{\lambda(r)} d r^{2}+\varphi(r) g_{a b}(\theta) d \theta^{a} d \theta^{b}
$$

where $g_{a b}(\theta)$ is a $G$-symmetric metric on $\partial K^{n}$. The functions $\nu(r), \lambda(r)$ and $\varphi(r)$ are found from the $4+n$-dimensional Einstein equations

$$
R_{A B}-\frac{1}{2} g_{A B} R+\Lambda g_{A B}=8 \pi G_{4+n} T_{A B}
$$

where $G_{4+n}, \Lambda$ are $4+n$-dimensional gravitational and cosmological constants and $T_{A B}$ is stress-energy tensor generated by the brane.

We are interested in dynamics of small perturbations $A_{\mu}^{a}$ around the background metric $g_{A B}^{b g}(2.6)$. In what follows we will analyze in detail the case of two extra dimensions when there is single angular coordinate $\theta$. The generalization on the case $n>2$ is straightforward.

\section{EQUATIONS OF MOTION FOR $A_{\mu}$.}

The Ricci tensor for the metric (2.6) perturbed by the fields $A_{\mu}(2.5)$ in the linear in $A_{\mu}$ approximation has the form

$$
\begin{aligned}
R_{\mu \nu}= & -e^{\nu-\lambda} \eta_{\mu \nu}\left(\frac{\nu^{\prime \prime}}{2}-\frac{\nu^{\prime} \lambda^{\prime}}{4}+\nu^{\prime 2}+\frac{\nu^{\prime} \varphi^{\prime}}{4 \varphi}\right)+\frac{1}{2}\left(\dot{A}_{\mu, \nu}+\dot{A}_{\nu, \mu}\right) \\
R_{\mu \theta}= & \frac{e^{-\nu} \varphi}{2}\left(A_{\nu, \mu \nu}-A_{\nu, \mu \mu}\right)+\frac{\varphi e^{-\lambda}}{2}\left[-A_{\mu}^{\prime \prime}+\left(\frac{\lambda^{\prime}}{2}-\nu^{\prime}-\frac{5 \varphi^{\prime}}{2}\right) A_{\mu}^{\prime}+\right. \\
& \left.\left(\frac{\varphi^{\prime 2}}{2 \varphi^{2}}-\frac{\varphi^{\prime \prime}}{\varphi}+\frac{\varphi^{\prime} \lambda^{\prime}}{2 \varphi}-\frac{\varphi^{\prime} \nu^{\prime}}{\varphi}\right) A_{\mu}\right] \\
R_{\mu r}= & \frac{1}{2 \varphi}\left(\varphi \dot{A}_{\mu}\right)^{\prime}-\frac{\nu^{\prime}}{2} \dot{A}_{\mu} \\
R_{r r}= & -2 \nu^{\prime \prime}-\nu^{\prime 2}+\nu^{\prime} \lambda^{\prime}+\frac{1}{2}\left(\frac{\varphi^{\prime 2}}{2 \varphi^{2}}+\frac{\varphi^{\prime} \lambda^{\prime}}{2 \varphi}-\frac{\varphi^{\prime \prime}}{\varphi}\right) \\
R_{r \theta}= & \frac{\varphi e^{-\nu}}{2} A_{\mu, \mu}^{\prime}
\end{aligned}
$$




$$
R_{\theta \theta}=\varphi e^{-\nu} \dot{A}_{\mu, \mu}+e^{-\lambda}\left(\frac{\varphi^{\prime} \lambda^{\prime}}{4}-\frac{\varphi^{\prime 2}}{4 \varphi}-\frac{\varphi^{\prime \prime}}{2}-\varphi^{\prime} \nu^{\prime}\right)
$$

When $A_{\mu}=0$ we get from (2.7), the expression of $\nu(r), \lambda(r)$ and $\varphi(r)$ through the brane stress-energy tensor $T_{A B}$. In the presence of $A_{\mu}$ the $\mu \nu, \mu r, r \theta$ and $\theta \theta$ components of Einstein equations (2.7) become

$$
\begin{aligned}
\dot{A}_{(\mu, \nu)} & =0 \\
\left(e^{-\nu} \varphi \dot{A}_{\mu}\right)^{\prime} & =0 \\
\dot{A}_{\mu, \mu}=A_{\mu, \mu}^{\prime} & =0
\end{aligned}
$$

Here prime denotes the derivative with respect to $r$ and dot denotes the $\theta$ derivative. The only nontrivial equation is the $\mu \theta$ component of Einstein equations. In the most simple case of flat extra dimensions when the background metric is

$$
d s^{2}=\eta_{\mu \nu} d x^{\mu} d x^{\nu}+d r^{2}+r^{2} d \theta^{2}
$$

it reduces to

$$
A_{\mu}^{\prime \prime}+\frac{5}{r} A_{\mu}^{\prime}+F_{\mu \nu, \nu}=0
$$

where we have denoted

$$
F_{\mu \nu}=A_{\mu, \nu}-A_{\nu, \mu}
$$

First of all we can see that the "zero mode" $A_{\mu}\left(x^{\nu}\right)$ which does not depend on $r, \theta$ and satisfies

$$
F_{\mu \nu, \nu}=0
$$

is a solution of the six-dimensional Einstein equations. The last system of equations is just the four-dimensional Maxwell equations on electromagnetic field $A_{\mu}(x)$. The $U(1)$ gauge group of electromagnetism is identified with the group $S O(2)$ of rotations of the normal bundle of the brane. Indeed, let us make a coordinate transformation

$$
\tilde{\theta}=\theta+v\left(x^{\mu}\right)
$$

which rotates normal vectors to the brane at the point $x^{\mu}$ on a small angle $v\left(x^{\mu}\right)$. The background metric (3.11) in new coordinates takes the form

$$
d s^{2}=\left(\eta_{\mu \nu}+r^{2} v_{, \mu} v_{, \nu}\right) d x^{\mu} d x^{\nu}+d r^{2}+r^{2} d \tilde{\theta}^{2}-2 r^{2} v_{, \mu} d x^{\mu} d \tilde{\theta}
$$

from where we see that in new coordinates

$$
A_{\mu}=-v_{, \mu}
$$

Since the metric (3.16) differs from (3.11) on coordinate transformation, it is again a solution of the Einstein equations. 
Obviously, by the same way of reasoning, the pure gauge configuration (3.17) must be a solution of the perturbed Einstein equations on a general background of the form (2.6). The $\mu \theta$ component of the Einstein equations in the general case is

$$
\left(e^{\nu-\lambda / 2} \varphi^{5 / 2} A_{\mu}^{\prime}\right)^{\prime}+e^{\lambda / 2} \varphi^{5 / 2} F_{\mu \nu, \nu}=0
$$

Taking the $\theta$ derivative of (3.18) and combining it with (3.9) we find that $A_{\mu}$ does not depend on $\theta$. Making a coordinate transformation (3.15) we can always fix the coordinate system in such a way that the condition

$$
A_{\mu, \mu}=0
$$

is satisfied on a particular surface $r=r_{0}$. Then, from (3.10) we conclude that the condition (3.19) remains valid for all $r$. The equation (3.18) in the gauge (3.19) reduces after the Fourier transform $\tilde{A}_{\mu}(p, r)=\int d^{4} x e^{-i p_{\nu} x^{\nu}} A_{\mu}(x, r)$ to

$$
\left(e^{\nu-\lambda / 2} \varphi^{5 / 2} \tilde{A}_{\mu}^{\prime}\right)^{\prime}+e^{\lambda / 2} \varphi^{5 / 2} m^{2} \tilde{A}_{\mu}=0
$$

where we have denoted $m^{2}=-p_{\mu} p^{\mu}$. This equation can be solved, for example, in the case of flat extra dimensions (3.11), $\lambda=\nu=0, \varphi=r^{2}$. The modes with $m^{2} \neq 0$ which are regular at $r=0$ have the form

$$
A_{\mu}=\frac{C}{r^{2}} J_{2}(m r)
$$

where $J_{2}(z)$ is the Bessel function and $C$ is an arbitrary constant.

Although the zero mode $A_{\mu}(x)$ is always a solution of Einstein equations locally, it can fail to be a global solution if the rotation invariance (3.15) is spontaneously broken. A simple example of this is when the flat extra dimensions (3.11) are compactified on two-dimensional torus $T^{2}$.

\section{LOCALIZATION OF $A_{\mu}$ ON THE BRANE.}

If the rotation symmetry (3.15) is not broken, the zero mode of the field $A_{\mu}$ is a global solution of higher-dimensional Einstein equations which, from the four-dimensional point of view, describes massless gauge field propagating along the brane. The equation (3.20) is a Strum-Liouville equation and the zero mode $A_{\mu}\left(x^{\nu}\right)$ is its normalizable solution in a space-time (2.6) which satisfies the condition

$$
N=\int d r e^{\lambda / 2} \varphi^{5 / 2}<\infty
$$

In such a space-time the massless field $A_{\mu}\left(x^{\nu}\right)$ mediates a Coulomb-like interaction between particles localized on the brane. For example, if we take a space-time with compact extra dimensions with topology of a sphere $S^{2}$, the bulk metric is

$$
d s^{2}=\eta_{\mu \nu} d x^{\mu} d x^{\nu}+\frac{1}{\left(1+r^{2} / a^{2}\right)^{2}} d r^{2}+\frac{r^{2}}{\left(1+r^{2} / a^{2}\right)^{2}} d \theta^{2}
$$


where $a$ is the radius of the sphere. This corresponds to

$$
\begin{aligned}
& \nu=0 \\
& \lambda=-2 \log \left(1+r^{2} / a^{2}\right) \\
& \varphi=r^{2} /\left(1+r^{2} / a^{2}\right)
\end{aligned}
$$

and the integral $N$ (4.1), obviously, converges.

Zero mode of the field $A_{\mu}$ can be normalizable also in space-times with noncompact extra dimensions. Let us take, for example, a space-time considered in [6]. This space-time is a solution of six-dimensional Einstein equations coupled to cylindrically symmetric magnetic field

$$
f_{r \theta}=B_{0} \sqrt{\varphi} e^{\lambda / 2-2 \nu}
$$

where $B_{0}$ is an arbitrary magnetic field strength. In the case when the cosmological constant in the bulk and on the brane are zero, the space-time metric is

$$
d s^{2}=\left(1+\frac{r^{2}}{a^{2}}\right)^{2 / 3}\left(\eta_{\mu \nu} d x^{\mu} d x^{\nu}+d r^{2}\right)+\frac{r^{2} d \theta^{2}}{\left(1+r^{2} / a^{2}\right)^{2}}
$$

where the parameter $a$ is related to the field strength (4.4)

$$
a^{2}=\frac{2}{3 \pi G_{6} B_{0}^{2}}
$$

The geometry of extra dimensions is schematically presented on Fig.1. The size $R_{\theta}$ of the circle parameterized by $\theta$ shrinks to zero as $r \rightarrow \infty$.

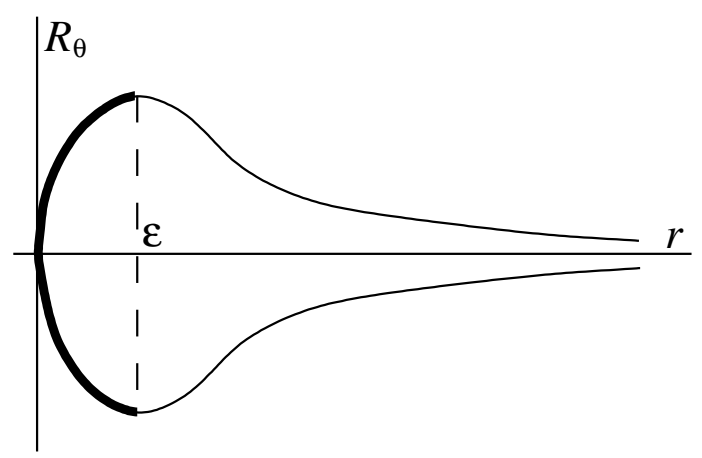

Figure 1: The geometry extra dimensions.

The integral $N$ (4.1) converges

$$
N=\int_{0}^{\infty} \frac{r^{5} d r}{\left(1+r^{2} / a^{2}\right)^{14 / 3}}=\frac{27 a^{6}}{440}
$$

and the zero mode $A_{\mu}\left(x^{\nu}\right)$ is a normalizable solution of (3.20) Note, that the volume of extra dimensions 


$$
V=\int \sqrt{-g} d r d \theta=\int\left(1+\frac{r^{2}}{a^{2}}\right)^{2 / 3} r d r d \theta
$$

is infinite so that the metric (4.5) does not localize gravity.

It is interesting to consider situation when both gravity and the gauge field are localized on the brane. This can be achieved in a six-dimensional generalization of Randall-Sundrum model which was considered in [21,22, 19]. The space-time is a solution of Einstein equations (2.7) with negative cosmological constant $\Lambda$. A string of finite size $\epsilon$ is placed at $r=0$ so that the stress-energy tensor $T_{A B}$ is different from zero in a small region $0 \leq r<\epsilon$ around $r=0$. Outside the string in the region $\epsilon<r<\infty$ the space-time metric is the six-dimensional anti-deSitter metric

$$
d s^{2}=e^{-c r} \eta_{\mu \nu} d x^{\mu} d x^{\nu}+d r^{2}+e^{-c r} R^{2} d \theta^{2}
$$

Here $R$ is an arbitrary constant which sets the size of the circle $S^{1}$ parameterized by $\theta$. Parameter $c$ is related to the bulk cosmological constant (2.7) as

$$
c=\sqrt{-\frac{2 \Lambda}{5}}
$$

The geometry of extra dimensions in this case is similar to that of space-time (4.5): the size of the circle $S^{1}$ parameterized by $\theta$ goes to zero as $r \rightarrow \infty$ (see Fig.1). The metric inside the brane is determined by the detailed structure of the brane stress-energy tensor $T_{A B}(r)$.

The equation (3.20) on $A_{\mu}$ reduces for the metric (4.9) to

$$
\left(e^{-7 c r / 2} \tilde{A}_{\mu}^{\prime}\right)^{\prime}+e^{-5 c r / 2} m^{2} \tilde{A}_{\mu}=0
$$

Its general solution in the gauge $(3.19)$ is

$$
\tilde{A}_{\mu}^{0}=e^{7 r / 4}\left(C_{1} J_{7 / 2}\left(\frac{2 m}{c} e^{c r / 2}\right)+C_{2} Y_{7 / 2}\left(\frac{2 m}{c} e^{c r / 2}\right)\right)
$$

The zero mode solution $A_{\mu}(x)$ of (4.11) is a normalizable solution of (4.11) since the integral $N(4.1)$ is converging

$$
N=\frac{2 R^{5}}{5 c}
$$

Note that the zero mode of $A_{\mu}$ is normalizable even in a more general space-time of "global string", considered in [19,23]

$$
d s^{2}=e^{-c_{1} r} \eta_{\mu \nu} d x^{\mu} d x^{\nu}+d r^{2}+e^{-c_{2} r} d \theta^{2}
$$

Here $c_{1}$ and $c_{2}$ are arbitrary positive constants related to the stress-energy tensor $T_{A B}(r)$ of the string which is nonzero even when $r \rightarrow \infty$. 


\section{MATTER FIELDS BOUND TO THE BRANE.}

The field $A_{\mu}$ interacts with matter fields bound to the brane. As an example we consider fermion fields propagating on the background (2.6).

Consider a six-dimensional Dirac spinor $\Psi$ which satisfies the equation

$$
\Gamma^{A} D_{A} \Psi=0
$$

The six-dimensional gamma matrixes $\Gamma^{A}$ are defined with the help of vielbein $E_{\hat{B}}^{A}$ and flat space gamma matrices $\Gamma^{\hat{A}}$

$$
\Gamma^{A}=E_{\hat{B}}^{A} \Gamma^{\hat{B}}
$$

(the indexes with hat are six-dimensional Lorenz indexes). The covariant derivative is defined as

$$
D_{A} \Psi=\Psi_{, A}+\frac{1}{2} \omega_{A}^{\hat{A} \hat{B}} \sigma_{\hat{A} \hat{B}} \Psi
$$

where $\omega_{A}^{\hat{B} \hat{C}}$ is the spin connection expressed through vielbein $E_{\hat{B}}^{A}$ and $\sigma_{\hat{B} \hat{C}}=\frac{1}{4}\left[\Gamma_{\hat{B}} \Gamma_{\hat{C}}\right]$. Taking the coordinate vielbein for the metric (2.6)

$$
\begin{aligned}
E_{\mu}^{\hat{\alpha}} & =e^{\nu / 2} \delta_{\mu}^{\hat{\alpha}} \\
E_{r}^{\hat{r}} & =e^{\lambda / 2} \\
E_{\theta}^{\hat{\theta}} & =\sqrt{\varphi}
\end{aligned}
$$

we find

$$
\begin{aligned}
& \omega_{\mu}^{\hat{A} \hat{B}} \sigma_{\hat{A} \hat{B}}=\frac{\nu^{\prime}}{2} e^{(\nu-\lambda) / 2} \Gamma_{\hat{\mu}} \Gamma_{\hat{r}} \\
& \omega_{\theta}^{\hat{A} \hat{B}} \sigma_{\hat{A} \hat{B}}=\frac{\varphi^{\prime}}{2 \sqrt{\varphi}} e^{-\lambda / 2} \Gamma_{\hat{\theta}} \Gamma_{\hat{r}}
\end{aligned}
$$

Let us consider the solutions of (5.1) which satisfy the condition

$$
\Gamma_{\hat{0}} \ldots \Gamma_{\hat{3}} \Gamma_{\hat{r}} \Gamma_{\hat{\theta}} \Psi=\Psi
$$

and can be presented in the form

$$
\Psi=e^{i q \theta} \chi(r) \psi\left(x^{\mu}\right)
$$

where $q$ is an integer. The two-component spinor $\chi(r)$ satisfies

$$
i \Gamma_{\hat{r}} \Gamma_{\hat{\theta}} \chi=-\chi
$$

while the four-component spinor $\psi(x)$ is chiral in four-dimensional sense

$$
i \Gamma_{\hat{0}} \ldots \Gamma_{\hat{3}} \psi=\psi
$$


and satisfies the four-dimensional massless Dirac equation

$$
\Gamma^{\hat{\mu}} \partial_{\mu} \psi=0
$$

From (5.1) we derive an equation on $\chi$

$$
\chi^{\prime}+\left(\nu^{\prime}+\frac{\varphi^{\prime}}{4 \varphi}+\frac{e^{\lambda / 2}}{\sqrt{\varphi}} q\right) \chi=0
$$

which is readily integrated

$$
\chi(r)=\text { const } \cdot \varphi^{-1 / 4} \exp \left\{-\nu-q \int_{r_{0}}^{r} d r^{\prime} \frac{e^{\lambda / 2}}{\sqrt{\varphi}}\right\}
$$

The solutions of (5.1) must be normalizable with respect to the norm

$$
\langle\Psi, \Psi\rangle=\int d^{4} x d r d \theta \sqrt{-g} \Psi^{*} \Psi=\int d r e^{2 \nu+\lambda / 2} \sqrt{\varphi}|\chi|^{2}
$$

In the case of space-time (4.9) the normalized solutions of equation (5.11) are given by

$$
\chi_{q}(r)=\frac{4 q^{3 / 2}}{R \sqrt{8 q^{2}+4 q c R+c^{2} R^{2}}} e^{5 c r / 4} \exp \left\{\frac{2 q}{c R}\left(1-e^{c r / 2}\right)\right\}
$$

The solution with $q=0$ with trivial dependence on $\theta$ grows at large $r$ and is not localized on the brane. But the solutions with $q>0$ decrease far from the brane. Here we have neglected a contribution from the region $0 \leq r<\epsilon$ inside the brane into the integral (5.13). In principle, the requirement of convergence of (5.13) in the limit $r \rightarrow 0$ will impose restrictions on the number of normalizable zero mode solutions of (5.1).

\section{COUPLING OF $A_{\mu}$ TO MATTER FIELDS.}

The fermion field $\Psi$ (5.1) produces a stress-energy tensor

$$
T_{A B}^{\Psi}=\frac{i}{2}\left(\bar{\Psi} \Gamma_{(A} D_{B)} \Psi-D_{(A} \bar{\Psi} \Gamma_{B)} \Psi\right)
$$

The $\mu \theta$ component of $T_{A B}^{\Psi}$ is

$$
\begin{aligned}
T_{\mu \theta}^{\Psi}= & \frac{i}{4} e^{\nu / 2}\left(\bar{\Psi} \Gamma_{\hat{\mu}} \Psi_{, \theta}-\bar{\Psi}_{, \theta} \Gamma_{\hat{\mu}} \Psi\right)+\frac{i \sqrt{\varphi}}{4}\left(\bar{\Psi} \Gamma_{\hat{\theta}} \Psi_{, \mu}-\bar{\Psi}_{, \mu} \Gamma_{\hat{\theta}} \Psi\right)+ \\
& \frac{i \sqrt{\varphi}}{4} e^{(\nu-\lambda) / 2}\left(\nu^{\prime}-\frac{\varphi^{\prime}}{\varphi}\right) \bar{\Psi} \Gamma_{\hat{\mu}} \Gamma_{\hat{r}} \Gamma_{\hat{\theta}} \Psi
\end{aligned}
$$

If we restrict our attention to the $\Psi$ configurations of the form (5.7), (5.8), (5.9), then (6.2) reduces to

$$
T_{\mu \theta}^{\Psi}=\frac{e^{\nu / 2}|\chi(r)|^{2}}{2}\left(m-e^{-\lambda / 2} \frac{\sqrt{\varphi}}{2}\left(\nu^{\prime}-\frac{\varphi^{\prime}}{\varphi}\right)\right) \bar{\psi} \Gamma_{\hat{\mu}} \psi
$$


We see that $T_{\mu \theta}^{\Psi}$ is proportional to the four-dimensional current

$$
j_{\mu}=\bar{\psi} \Gamma_{\hat{\mu}} \psi
$$

Substituting the stress-energy tensor (6.3) into the Einstein equations (2.7) we find that equation (3.20) on $A_{\mu}$ is modified

$$
\left(e^{\nu-\lambda / 2} \varphi^{5 / 2} \tilde{A}_{\mu}^{\prime}\right)^{\prime}+e^{\lambda / 2} \varphi^{5 / 2} m^{2} \tilde{A}_{\mu}=-8 \pi G_{6} e^{2 \nu+\lambda / 2} \varphi^{3 / 2}|\chi|^{2} Q \tilde{j}_{\mu}
$$

where $\tilde{j}_{\mu}$ is the Fourier transform $\tilde{j}_{\mu}(p, r)=\int d^{4} p e^{-i p_{\mu} x^{\mu}} j_{\mu}(x, r)$ of the four-dimensional current. We also have denoted

$$
Q=q-e^{-\lambda / 2} \frac{\sqrt{\varphi}}{2}\left(\nu^{\prime}-\frac{\varphi^{\prime}}{\varphi}\right)
$$

We can write a solution of this equation in terms of a Green function $\tilde{\Delta}\left(p, r, r^{\prime}\right)$

$$
\tilde{A}_{\mu}=-8 \pi G_{6} \tilde{j}_{\mu} \int d r^{\prime} \tilde{\Delta}\left(p, r, r^{\prime}\right) Q e^{2 \nu+\lambda / 2} \varphi^{3 / 2}|\chi|^{2}
$$

The Green function of equation (6.5) is expressed in terms of normalized solutions $A_{m}^{0}(r)$ of homogeneous equation (3.20) for a fixed $m^{2}$

$$
\tilde{\Delta}\left(p, r, r^{\prime}\right)=\frac{1}{N p^{2}}+\sum_{m^{2}>0} \frac{A_{m}^{0}(r) A_{m}^{0}\left(r^{\prime}\right)}{p^{2}+m^{2}}
$$

where $N$ is the normalization constant (4.1). If we are interested in the behavior of $A_{\mu}$ at large distances from the source, we can restrict attention to the limit $p^{2} \rightarrow 0$ in which zero mode solution of (3.20) gives the leading contribution into the Green function $\tilde{\Delta}$. In this limit

$$
\tilde{A}_{\mu} \approx \frac{8 \pi G_{6}}{N} \frac{\tilde{j}_{\mu}(p)}{p^{2}} \int d r^{\prime} Q \varphi^{3 / 2} e^{2 \nu+\lambda / 2}\left|\chi\left(r^{\prime}\right)\right|^{2}
$$

In the case of asymptotically anti-deSitter space-time (4.9) the normalization constant $N$ is given by (4.13) while the charged zero modes of Dirac field have the radial profile $\chi(r)$ given by (5.14). In the limit

$$
c R \ll 1
$$

when the curvature radius $\rho=c^{-1}$ of anti-deSitter space is much larger than the thickness of the brane $R$, the expression (6.9) for $\tilde{A}_{\mu}$ reduces to

$$
\tilde{A}_{\mu}=\frac{20 \pi c G_{6}}{R^{3}} \frac{\left(q \tilde{j}_{\mu}\right)}{p^{2}}
$$

Comparing the last equation to the standard expression of the four-dimensional Maxwell theory we find the effective fine structure constant 


$$
\alpha=\frac{5 c G_{6}}{R^{3}}
$$

From (5.14) we can see that the fermion zero modes with nonzero charge $q$ are localized in a region with a size of order of the brane thickness $R$. If $R$ is small enough we can approximate the profile $\chi(r)$ by delta function

$$
|\chi|^{2} \approx \frac{1}{R} \delta(r)
$$

If we are interested in interactions of particles localized on the brane we need only the expression for the Green function $\tilde{\Delta}$ at $r, r^{\prime}=0$. The exact expression for $\tilde{\Delta}$ can be found in a way similar to the one used in [24] for the calculation of the Green function for gravitational perturbations of Randall-Sundrum model. For $r, r^{\prime}=0$ we get

$$
\tilde{\Delta}(p)=\frac{5 c}{2 k^{2}}-\frac{1}{k} \frac{H_{3 / 2}^{(1)}(2 k / c)}{H_{5 / 2}^{(1)}(2 k / c)}
$$

where $k^{2}=-p^{2}$. Expressing the Hankel functions $H_{3 / 2}^{(1)}(z)$ and $H_{5 / 2}^{(1)}(z)$ through the elementary functions we get for $A_{\mu}$

$$
\tilde{A}_{\mu}=\frac{8 \pi G_{6}}{R^{3}}\left(\frac{5 c}{2 k^{2}}+\frac{2(c-2 i k)}{4 k^{2}+6 i c k-3 c^{2}}\right) q \tilde{j}_{\mu}
$$

In the case of static configurations $p_{0}=0$ the first term in the last equation gives the conventional Coulomb law at large distances from a static source. The second term of (6.15) results in corrections of order of $O\left(1 / L^{3}\right)$ to Coulomb law at distances $L \sim c^{-1}$ along the brane.

\section{CONCLUSION.}

We have considered models in which a brane universe $M^{4}$ is embedded into a space-time $\mathcal{M}^{6}$ with $n=2$ warped infinite extra dimensions. The Kaluza-Klein field $A_{\mu}$ associated to the $U(1)$ group of rotations of the normal bundle of $M^{4}$ is localized on the brane if the background metric (2.6) is such that the integral $N$ (4.1) is converging. In the case of asymptotically anti-deSitter metric in six-dimensional bulk the fermion zero modes which possess nonzero rotation moment $q$ around the brane are localized on $M^{4}$ (see (5.14)). They are charged with respect to $A_{\mu}$ and $A_{\mu}$ mediates Coulomb-like interaction between these zero modes. We have calculated the effective four-dimensional fine structure constant $\alpha$ (6.12) in such a model. It is expressed through the inverse curvature radius $c$ of the bulk antideSitter space and the brane thickness $R$. The presence of infinite extra dimensions results in modification of the four-dimensional photon propagator and in power-law corrections to the Coulomb law at the distances of order of the inverse curvature radius $c$ of anti-deSitter space (6.15). 


\section{ACKNOWLEDGEMENT.}

I am grateful to A.O.Barvinsky, V.F.Mukhanov, V.A.Rubakov, I.Sachs, and S.Solodukhin for fruitful discussions of the subject of the paper. This work was supported by SFB 375 der Deutschen Forschungsgemeinschaft. 


\section{REFERENCES}

[1] T.Appelquist, A.Chodos, P.G.O.Freund, eds., Modern Kaluza-Klein theories, AddisonWesley, 1987

[2] E.Witten, "Fermion quantum numbers in Kaluza-Klein theory", in [1], p. 438

[3] S.Randjbar-Daemi, A.Salam, J.Strathdee, Nucl. Phys. B 214, 491 (1983)

[4] C.Wetterich, Nucl. Phys. B 255, 480 (1985)

[5] V. A. Rubakov and M. E. Shaposhnikov, Phys. Lett. B125, 136 (1983).

[6] G. W. Gibbons and D. L. Wiltshire, Nucl. Phys. B287, 717 (1987).

[7] N. Arkani-Hamed, S. Dimopoulos and G. Dvali, Phys. Rev. D 59, 086004 (1999) hepph/9807344.

[8] L. Randall and R. Sundrum, Phys. Rev. Lett. 83, 4690 (1999) hep-th/9906064.

[9] T. Han, J. D. Lykken and R. Zhang, Phys. Rev. D 59, 105006 (1999) hep-ph/9811350.

[10] S. Cullen and M. Perelstein, Phys. Rev. Lett. 83, 268 (1999) hep-ph/9903422

[11] A. Neronov, Phys. Lett. B472, 273 (2000) gr-qc/9911122]

[12] A. Neronov and I. Sachs, "On metric perturbations in brane-world scenarios," hepth/0011254.

[13] N. Arkani-Hamed and M. Schmaltz, Phys. Rev. D 61, 033005 (2000) hep-ph/9903417.

[14] A. Neronov, "Brane world cosmological constant in the models with large extra dimensions," gr-qc/0101060.

[15] I. Antoniadis, C. Munoz and M. Quiros, Nucl. Phys. B397, 515 (1993) hepph/9211309].

[16] R. Jackiw and C. Rebbi, Phys. Rev. D 13, 3398 (1976).

[17] G. Dvali and M. Shifman, Phys. Lett. B396, 64 (1997) hep-th/9612128.

[18] S. L. Dubovsky, V. A. Rubakov and P. G. Tinyakov, JHEP0008, 041 (2000) hepph/0007179.

[19] I. Oda, Phys. Lett. B496, 113 (2000) hep-th/0006203.

[20] G. Dvali, G. Gabadadze and M. Shifman, Phys. Lett. B497, 271 (2001) hepth/0010071.

[21] R. Gregory, Phys. Rev. Lett. 84, 2564 (2000) hep-th/9911015.

[22] T. Gherghetta and M. Shaposhnikov, Phys. Rev. Lett. 85, 240 (2000) hep-th/0004014.

[23] T. Gherghetta, E. Roessl and M. Shaposhnikov, Phys. Lett. B491, 353 (2000) hepth/0006251].

[24] S. B. Giddings, E. Katz and L. Randall, JHEP0003, 023 (2000) hep-th/0002091. 